\title{
Free-Hand versus Surgical Guide Implant Placement
}

\author{
Aysooda Afshari $\mathbb{D}^{1},{ }^{1}$ Rojin Shahmohammadi, ${ }^{2}$ Seyed Ali Mosaddad ${ }^{D},{ }^{3}$ Ozra Pesteei $\left(\mathbb{D},{ }^{2}\right.$ \\ Emran Hajmohammadi $\mathbb{D}^{4},{ }^{4}$ Mahdi Rahbar $\mathbb{D}^{\mathbb{D}},{ }^{5}$ Mostafa Alam $\mathbb{D}^{6},{ }^{6}$ and Kamyar Abbasi $\mathbb{D}^{7}$ \\ ${ }^{1}$ Postgraduate Student of Prosthodontics, School of Dentistry, Tehran University of Medical Sciences, Tehran, Iran \\ ${ }^{2}$ Postgraduate Student of Periodontics, School of Dentistry, Tehran University of Medical Sciences, Tehran, Iran \\ ${ }^{3}$ Student Research Committee, School of Dentistry, Shiraz University of Medical Sciences, Shiraz, Iran \\ ${ }^{4}$ Department of Oral and Maxillofacial Surgery, School of Dentistry, Ardabil University of Medical Sciences, Ardabil, Iran \\ ${ }^{5}$ Department of Restorative Dentistry, School of Dentistry, Ardabil University of Medical Sciences, Ardabil, Iran \\ ${ }^{6}$ Department of Oral and Maxillofacial Surgery, School of Dentistry, Shahid Beheshti University of Medical Sciences, Tehran, Iran \\ ${ }^{7}$ Department of Prosthodontics, School of Dentistry, Shahid Beheshti University of Medical Sciences, Tehran, Iran
}

Correspondence should be addressed to Ozra Pesteei; ozra.pesteei@gmail.com

Received 16 December 2021; Accepted 30 December 2021; Published 17 February 2022

Academic Editor: Hamid Tebyaniyan

Copyright (c) 2022 Aysooda Afshari et al. This is an open access article distributed under the Creative Commons Attribution License, which permits unrestricted use, distribution, and reproduction in any medium, provided the original work is properly cited.

\begin{abstract}
One of the most key areas of dentistry is dental implant surgery. The use of digital equipment and software in dentistry has developed considerably in recent years compared to other fields of medicine. Since examining the advantages and disadvantages of each approach, along with case studies, can help physicians make informed decisions, this review study aims to raise the awareness of dentists to make easier decisions about using guided or free-hand surgery. When planning for a dental implant, one of the most challenging questions that doctors face is which method to use (guided surgery or free-hand). Choosing the right method, such as other clinical considerations, will depend on the individual circumstances of each patient and the preference of the treating physician. Free-hand surgery is a cost-effective method in which the flap is reflected, and, according to the doctor's diagnostic information, an implant is placed, which in many cases is a useful method. Guided surgery has the highest level of accuracy and control, in which osteotomy is designed and printed through a digital surgery guide, and depending on the complexity of the case and the patient's anatomy, it has a higher level of value than free surgery. The surgical guide helps the surgeon make the implant surgery more accurate, safer, simpler, at a lower cost, and in less time. In fact, there are patterns that convey information about the position of the tooth to the dentist before the implant is placed.
\end{abstract}

\section{Introduction}

Dental implants are performed when a person has lost a tooth for any reason, and the implant is used to fill in the gaps [1]. One of the key factors that make implants known as a reliable option is successful osseointegration, which requires a method that achieves minimized surgical complications such as nerve damage, perforation, and cortical plate perforation to achieve this goal and ultimately achieve the desired result [2]. One of the key factors that make implants known as a reliable option is successful osseointegration, which requires a method that achieves minimized surgical complications such as nerve damage, perforation, and cortical plate perforation to achieve this goal and ultimately achieve the desired result [3]. The most common clinicalpathological findings associated with dental implants are hard tissue defects [4], such as defects at implant sites encompass intra-alveolar [5], dehiscence, fenestration, horizontal ridge [6], and vertical ridge defects and soft-tissue defects include volume and quality deficiencies with a lack of keratinized tissue [7], which can lead to marginal bone loss, soft-tissue inflammation, and soft-tissue stagnation [8-10].

Since the introduction of modern implantology to the medical community in the early 1980s, surgeons have always sought to place implants in terms of the amount of bone left in the patient's jaw [11]. This sometimes causes the implants to be placed in the wrong direction inside the jaw and, in many cases, makes it difficult or impossible to achieve a 
proper prosthesis, both aesthetically and functionally [12]. It is important to understand that surgeons usually tend to place the implants in the largest volume of bone left, but in most cases, this shape of the implant has caused the buccal or lingual position of the implant to be too much, and it provides problems for prosthodontists and laboratory technicians in preparing prostheses as efficiently and beautifully as possible [13]. Unfortunately, such problems remain hidden from the surgeon and patient until the implant is cast, and the prosthodontist uses very expensive equipment and unusual prostheses to treat the patient, which, although it leads to the preparation of prostheses, however, in the end, despite all the efforts of the prosthetic staff, is inefficient and ugly for the patient and sometimes causes legal issues which, of course, pleases no one [14]. In this regard, the use of new technologies and modern software provides the possibility of three-dimensional examination of the location of implants, making the diagnosis and treatment of patients more reliable [15].

Dental implants are performed in two general ways, which are implantation with the normal method and implantation with the surgical guide. The surgical guide allows dental implants to be performed in the most accurate location and with the least amount of surgery. The correct position of the implant allows the optimal design of the definitive prosthesis and prevents the possibility of cemented repairs by making it possible to design and manufacture recyclable screw prostheses $[10,16]$. From the patient's point of view, guided surgical procedures are no different from conventional surgery, and only an additional scan/image is taken of the patient's mouth. The procedure is that the teeth scan and CBCT of the patient's jaw are merged together in the guide design software [17]. As a result, the nerves of the jaw, sinuses, the roots of adjacent teeth, and bone density are examined before each operation, and the implant is placed virtually in the best location [18].

Based on this design, a surgical guide is made and sent to the treating dentist. It is even possible for all veneers to be sent to the dentist before the patient visits. Therefore, the dentist places the implant in the same space as specified in software without stress and, more importantly, without error [4]. As a result, the patient in one session with the lowest risk of infection will pass the implantation stages without surgery and with the lowest risks [19]. In some cases, a combination of surgery and a surgical guide is used as directed by a physician. If the implant can be placed in the best place and in the best conditions, it will lead to a long life of the prosthesis [20]. Despite the fact that different studies have introduced different types of these surgical guides, it does not seem to be any strong consensus either on classification or on defining different types of surgical guides $[21,22]$; therefore, the aim of this review study was to gather available data in regard to the classification and the whole idea of practicing implant dentistry using guided surgery.

\section{Free-Hand Surgery vs. Guided Surgery}

In free-hand surgery, panoramic and periapical radiographs are used to assess the width and alveolar bone profile available to place the implant and examine the surrounding anatomy and ultimately rely on $\mathrm{CBCT}$ imaging. In this method, periodontal probes, gauges, or calipers are applied through the intraoral exam to make a sound in the bone, which gives a logical view of the height and thickness of the ridge [23]. The surrounding teeth can also be used as a guide for determining the correct position of the implant. It should be noted that the implant should be at least $1.5 \mathrm{~mm}$ from each adjacent tooth and $2 \mathrm{~mm}$ apical to their enamel cementoenamel junction [13].

Implants that are placed too close to the root of an adjacent tooth can result from poor surgical technique, poor treatment planning, insufficient space, and incorrect angle, which can damage the periodontal ligament and surrounding structures. This can lead to bone displacement in the periodontal ligament (PDL) space, which ultimately leads to changes in blood supply to adjacent teeth, loss of tooth freshness, apical periodontitis, and internal or external resorption [24].

Implants very close to nearby teeth are more likely to be lost due to infection or bone resorption. If the distance between the implant is more than $1.5 \mathrm{~mm}$ to the adjacent tooth, any bone defect around the implant remains a vertical defect which in most cases; the bone loss does not occur in adjacent natural teeth, and if this distance is less than $1.5 \mathrm{~mm}$, then the bone on the adjacent tooth will maintain the height of the interdental papilla. In any case, not observing enough space between the tooth and the implant can lead to irreparable damage to adjacent teeth and their decay and fracture [13].

Free-hand surgery has many benefits for the dentist because it can visualize and relate diagnostic data to the actual clinical condition by reflecting soft tissues and examining bone anatomy [25]. Additional treatments such as bone grafts, PRF, and GBR can be easily performed if needed. On the contrary, the surgeon is able to make measurements through diagnostic casts, and making a diagnostic replica model gains a better understanding of the mesial-distal space and the apico-coronal space in order to determine the exact location of the implant on adjacent teeth by the prosthesis [26]. The use of diagnostic wax will also help in planning the surgical procedure, which will result in the implant supporting the final prosthesis in the best possible way. In free-hand surgery, the bone beneath the implant can be directly evaluated during surgery and measured with bone calipers after opening the flap [23].

The procedure for guided implant surgery is different from free-hand implant surgery. In guided implant surgery, after completing the CBCT imaging, a DICOM file is created, which is accompanied by a digital intraoral impression or precise putty light body impression to prepare the model. The DICOM file is passed to the implant scheduling software along with the patient data. This software uses DICOM file to present data in two dimensions and three dimensions $[10,27]$. Figure 1 demonstrates conventional and digital workflow as a chart [28].

Software allows the user to visualize important anatomical milestones such as the nasopalatine canal, maxillary sinus, inferior alveolar canal, and submandibular fossa, which is an auxiliary factor in implant position planning using different tools [29]. 

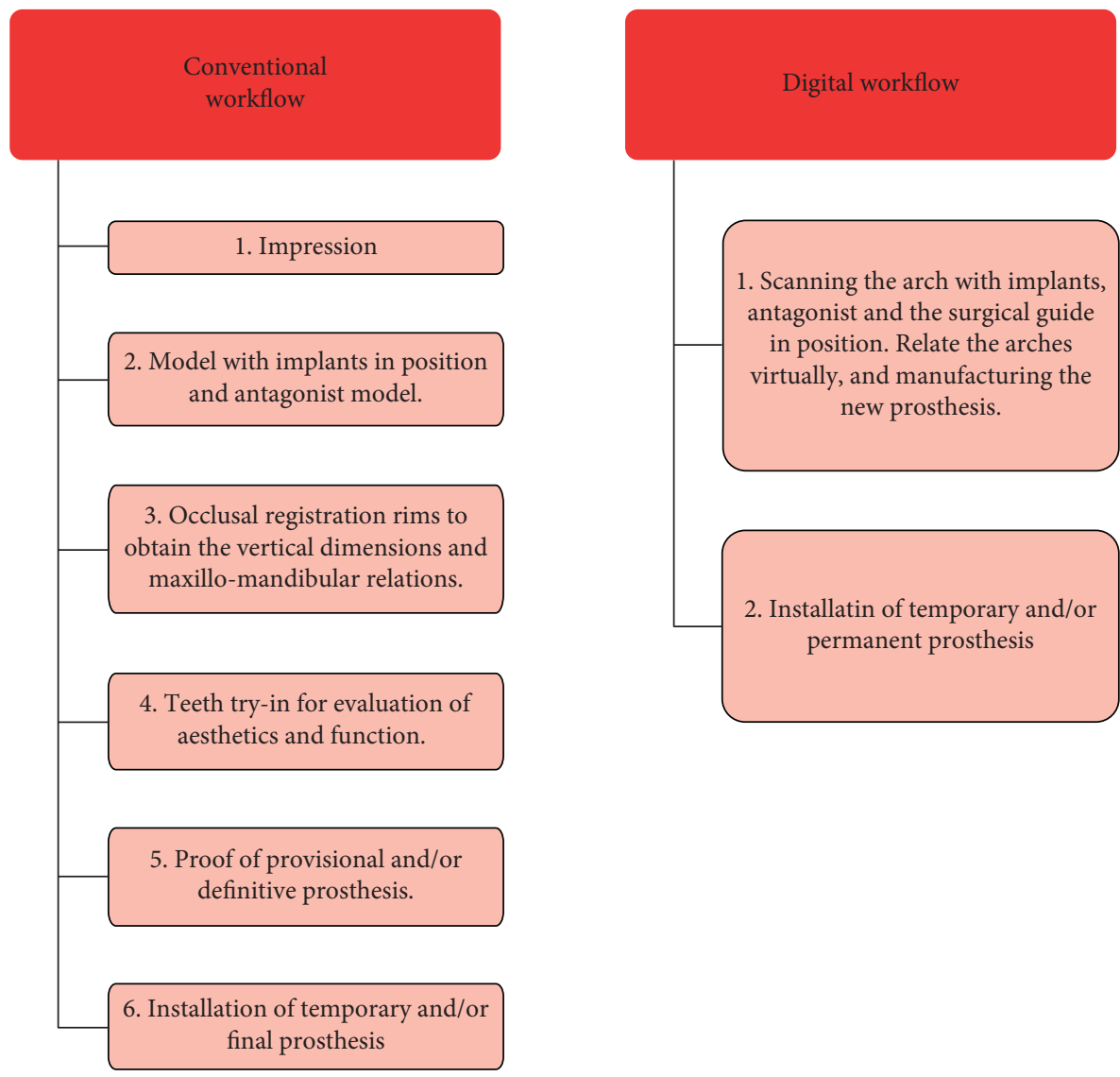

FIGURE 1: Conventional versus digital workflow in implantology [28].

After completing the treatment planning, the obtained digital information is included in the stereotyped surgery guide and becomes physically available. The surgery guide pattern can be designed differently depending on whether the patient has teeth or is completely toothless, depending on the number of implants to be implanted. For example, in patients whose teeth are preserved around the implant site, the surgical guide pattern is used to maintain stability [30]. In toothless people, the pattern has horizontal sleeves for anchor pins to be fixed in the patient's mouth [31].

This guide has round metal sleeves made of titanium or other alloys at the implant site that the depth, angulation, and mesial-distal and buccal-lingual locations of the implant are precisely controlled by these titanium sleeves [32]. Each surgery guide is made according to the guided implant surgery kit. The drills in this kit are designed to be suitable for osteotomy and suitable to perform it. Each of any drills has a stopper that rests on the occlusal exterior of the lip of the metal sleeves. The length of the metal sleeves determines the distance between the neck of the implant and the occlusal surface of the sleeves (offset) [31].

\section{Classification of Surgical Guides}

There are different opinions about the classification of several types of surgical guides. For example, Balshi and
Garver [33] consider the condition of the patient's teeth as the main parameter and introduce three basic surgical guide stents for implant placement.

(1) Completely edentulous (supplies a general guide to the area of dental implant positioning and a particular guide to the location and angulation that of each dental implant have need of placement) (Figure 2) [34]

(2) Slightly edentulous/removable partial denture design

(3) Slightly edentulous tooth-supported design (Figure 3) [35]

Considering the remaining dentition and needed accuracy for the guide, there have proposed 4 options in regard to supporting area: tooth supported, tissue supported, tooth and tissue supported, and tissue supported with an accessory fixation for edentulous patients [36].

A conceptual method is also used to describe different types of surgical guides, which includes three different concepts [24, 37]:

(1) Nonlimiting design

(2) Partially limiting design

(3) Completely limiting design

These three concepts are classified based on the amount of limitation that surgical guide templates offer [37]. 


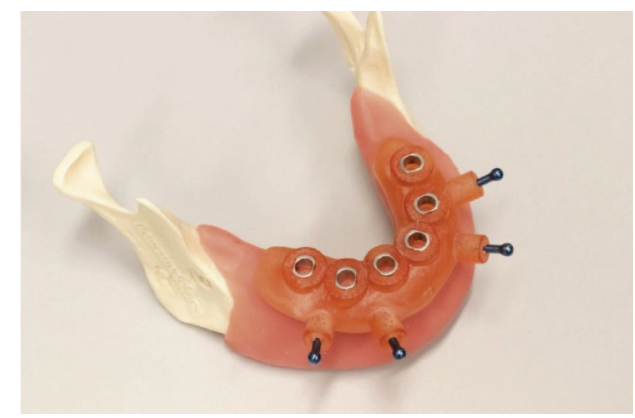

FIgure 2: Printed surgical guide for a full mouth rehabilitation using four guide pins [34].

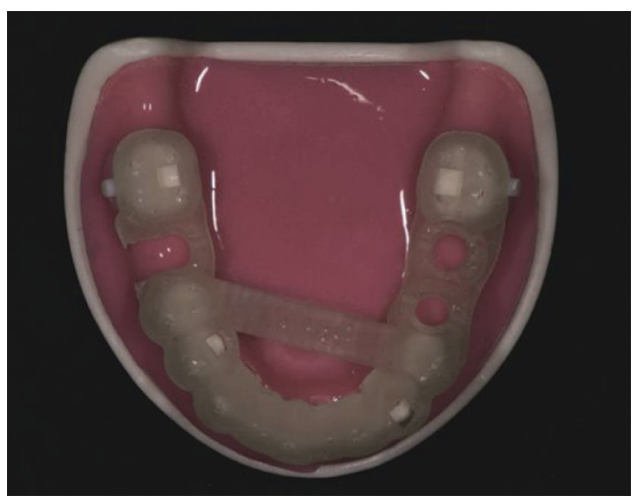

(a)

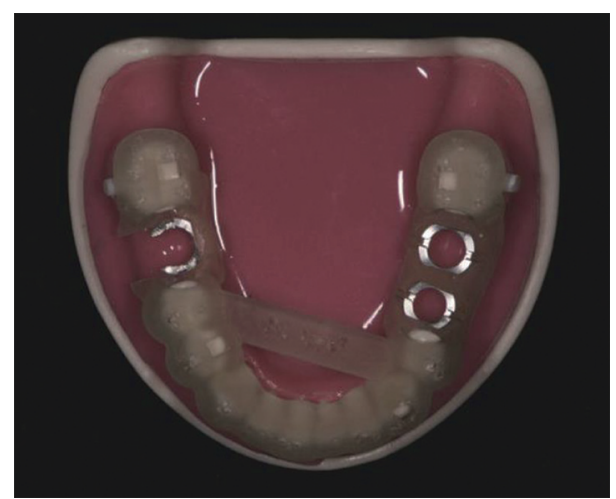

(b)

FIgURE 3: (a) Metal sleeve-free surgical guide. (b) Metal sleeve incorporated surgical guide [35].

The first one is a simple, unrestricted surgical guide, socalled free guide [36], that tells the operator where to go in relation to the implant site and guide the surgeon on where the future prosthesis would be in relation to the implant, and the operator will decide on other parameters related to the exact angle and position of the implant. It also provides the surgeon with the best location of the implants without too much focus on the angulation of the surgical drill, therefore allowing too much flexibility; the operator will decide on other parameters related to the exact angle and final position of the implant. In this regard, a technique was described in studies $[38,39]$ in which a guide pin hole is fabricated by drilling through a clear vacuum-formed matrix. This hole functioned as the best position of the implant and adjacent and opposing teeth were used as a guide to determine the angulation. Therefore, these templates could be served as imaging indicators in the implant surgical phase.

The second type of surgical guide known as access guide [36] is somewhat restrictive, which is a guide sleeve that will direct only the first drill used for the preparation of the implant site there, and other preparation steps are done by the surgeon [37].

As the last concept, the completely restrictive surgical guide or the precision guide [36] wherein all the instruments used for drilling the implant area by that guide, containing the buccolingual and mesiodistal planes, as well as to drill, stops limiting the depth of the preparation and so the final positioning of the prosthetic part [37]. Since these guides are more restrictive, decision-making and following surgical procedure that is done during the operation would be less. This concept has 2 common designs: computer-assisted design and manufacturing (CAD/CAM)-based surgical guide and cast-based guided surgical guides.

Considering 3 design concepts and 4 supporting area mentioned before, there could be 12 types of surgical guides [36]. Requiring a free or access guide with tissue support and accessory fixation would be somehow rare; therefore, feasible options for a surgical guide would be as follows:

(1) Nonlimiting, tooth supported

(2) Nonlimiting, tooth and tissue supported

(3) Nonlimiting, tissue supported

(4) Partially limiting, tooth supported

(5) Partially limiting, tooth, and tissue supported

(6) Partially limiting, tissue supported

(7) Completely limiting, tooth supported

(8) Completely limiting, tooth, and tissue supported

(9) Completely limiting, tissue supported

(10) Completely limiting, tissue supported with accessory fixation

\section{The Effect of Surgical Guide Pattern on Implant Accuracy}

Accurate placement of the implant is critical to achieving an aesthetic result and correct alignment to withstand occlusal 
forces for long-term success [40]. Despite the popularity of the surgical guide model in the clinic, there is still disagreement about its effect on the accuracy of implant placement. Some researchers believe that implant placement using a surgical guide is more accurate than other methods [23]. Others have argued that despite the high accuracy of dental implants when using the surgical guide, free-hand implant surgery accuracy has been sufficient and acceptable for most clinical conditions [8].

In their study, Wang et al. showed that the use of the surgical guide makes a significant difference between the planned and actual positions of the implant, especially at the implant shoulder, root apex, and angulation relative to the manual implantation method [41]. It has been clearly proven that the placement of dental implants using surgical guides is more accurate than implants that are placed without a guide $[42,43]$. High accuracy in implant placement is of great clinical importance and has several benefits. Among these benefits is its safety. Preoperative planning using surgical guides can ensure the safety of implant placement and reduce the incidence of complications, which is an important advantage for young and inexperienced surgeons and makes implant surgery easier for them. Also, when planning for a surgical guide, it is easy to evaluate that the proper angulation and occlusal relationships are more readily assessable using dental casts where the lingual aspect is not obscured $[44,45]$.

\section{Evaluating the Effect of Accuracy Factors on the Position and Angle of the Implant}

Determining the exact position of the implant in the bone is often difficult due to the location of the implant and its angle $[46,47]$. Physician experience, tooth-born status, timing relative to extraction, and the number of adjacent implants are identified as the four major factors influencing implant position. Also, tooth-borne status, number of adjacent implants, and the width of the edentulous space for the subset of tooth-borne, single-implant cases, have been introduced as three main influential factors on implant angulation [13].

It has been observed that the number of implants that are to be placed in proximity has a significant effect on the position of the implant and its angle. As mentioned, singleimplant cases are more accurate than cases where 2 or 3 implants are placed side by side [48]. Also, the angle and position of implants placed in people who have teeth are more accurate than in people who are completely toothless. Among the factors influencing accuracy, the presence of adjacent teeth on both sides of the implant has the greatest impact on the accuracy of position. The location of the extracted tooth is also one of the factors affecting the accuracy of the implant angle, but its impact is less than the impact of the number of adjacent implants [49].

Another influential factor is the time of implant placement relative to the time of tooth extraction. Delayed implants (time interval between tooth extraction to implant placement) have been observed to be significantly less accurate and the cases of immediate implants (implantation done on the same day as the tooth is extracted) are in a more precise position [50]. In terms of mesiodistal angulation between immediate and delayed cases, no significant statistics have been obtained [51]. The results of various studies show that immediate implants are in a more accurate mesiodistal position, but do not differ in angle [52].

The most severe angular differences in the lower-molar implant subset have been observed in cases with distinct radiographic lines from the previously extracted tooth. In summary incomplete, partial radiographic bone remodeling after extraction predicted higher positioning accuracy and a trend toward lower angulation accuracy. It is also reported that arch, location on the arch, and implant dimensions have little to do with planting accuracy. In general, the results of studies show that fully guided implants that use a surgical guide are more accurate than implants that use traditional surgical guides and free-hand surgeries [44, 52-54]. Figure 4 [34] depicts the accuracy obtained through guided surgery by superimposition of the digitally planned implants and the actual treatment.

\section{The Rate of Failure Associated with Implant Placement by Surgical Guides versus Free- Hand}

The amount of research carried out on the impact of the surgical guide on implant success and survival is limited. Criteria for implant success include peri-implant radiolucency, the absence of mobility, pain, and infection $[55,56]$. It was stated that the the maximum annual bone loss after one year of implant placement should be less than $0.2 \mathrm{~mm}[55]$.

Implant survival can be tracked by examining its stability in post-implant examinations. It is stated that the standard success rate should be $85 \%$ after 5 years of implant placement and $80 \%$ after 10 years [57-60]. Primary implant failure, which is mainly due to improper planning or surgical complications, causes problems for patients and surgeons [61, 62]. Implanted sites that fail for the first time are less likely to survive and are more at risk if they are re-implanted $[63,64]$. Therefore, efforts should be made to make the initial implant successful. The success and survival of the implant depends on the osseointegration between the surface of the implant and the bone around the implant site [65] so that the loss of bone formation will cause the implant to fail $[66,67]$. Most of the initial implant failures occur before the prosthesis is loaded, which is seen in less than $5 \%$ of patients in the first few weeks $[68,69]$. Late failures that can be due to factors, such as circumstances affecting the microbial environment, peri-implantitis, and prosthetic rehabilitation, occur most often after the prosthesis is loaded. These failures are seen in an average of $7 \%$ of patients [70-72].

In a study, Yogui examined the survival rate of implant placement in both surgical guides and free-hand methods. The results of his 5-year study on implant survival in patients show that both techniques have had similar results in terms of survival, with rates ranging from 91 to $100 \%$ [25]. In another systematic study, the rate of implant failure in free- 


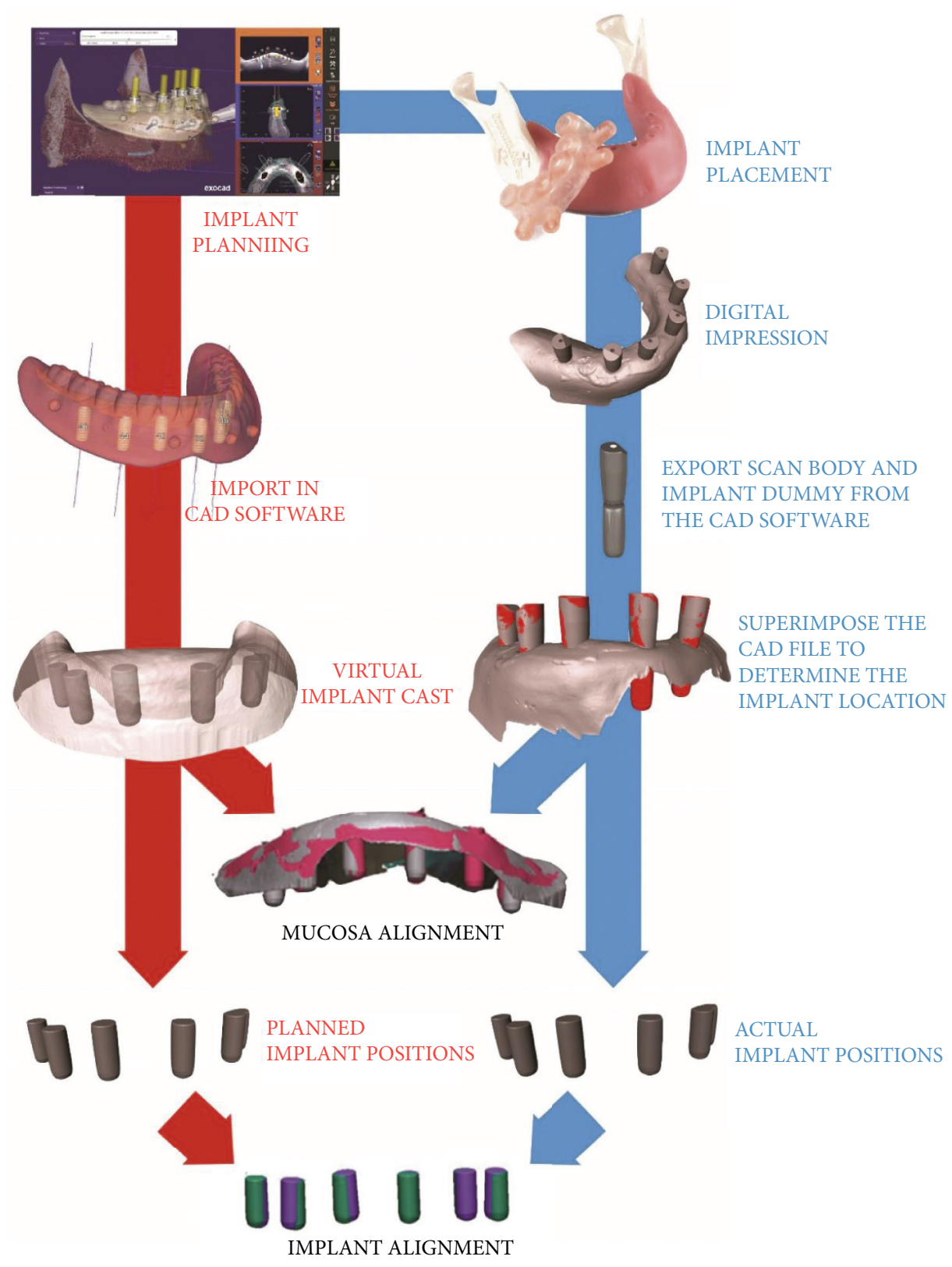

FIgURE 4: Actual and planned treatment plan superimposition regarding accuracy [34].

hand was reported to be almost three times that of surgical guides [73]. In addition to the type of implant implantation, factors which can increase the initial and late risk of implant failure include age and sex [74], smoking [56], tooth loss, bone quality and volume [75], implant site, diameter and length of the site [76], and, finally, the immune factor and various systemic diseases [56].

\section{Complications and Possible Errors of Guided Implant Surgery}

The risks of guided implant surgery are related to possible deviations between the position of the planned implant and the final clinical outcome. Accuracy can be achieved using specialized software. Accuracy check is possible with parameters such as deviation at the entry point, deviation at the apex, angle deviation or deviation of the long axis, and deviation in height/depth [6]. On an average, $<0.5 \mathrm{~mm}$ errors were reported from computed tomography or conebeam computed tomography imaging acquisition and data processing [77, 78].

Recently, cone-beam computed tomography has been preferred to multilayer computed tomography for implant treatment planning due to its relatively low radiation dose, lower cost, high-resolution 3D images, and higher operating speed $[79,80]$. Another cause of implant-guided surgery error is the patient's movement during the scan. Therefore, using an occlusal bite index to stabilize the mandible and scan the prosthesis, especially in patients who do not have teeth, will help to correct the error, and the scan should be repeated if necessary [81]. 


\section{Results of Guided Implant Surgery Compared to Free-Hand Implant Placement}

In various studies, successful use of guided implant surgery has been reported in people who have completely lost their teeth for any reason, along with the concomitant delivery of a prefabricated prosthesis for immediate replacement of missing teeth [82]. Nickenig et al. used guided implant surgery on patients who were partially edentulous, with $58 \%$ of the 250 implants performed on 102 patients without flaps. An implant which is planned had to be converted to a shorter one, and in four next circumstances, the limited interocclusal distance impersonated challenges while drilling. In eight cases, the guide could not be used, and implant placement required bone strengthening, which delayed implant placement. In nine of the implants, the final angle was different from the planned angle, with no clinical consequences [54].

In another study, which was performed comparatively between two groups of patients, it was observed that, in flapless patients, the duration of surgery, pain intensity and analgesia, and cases of trismus and bleeding were much lower than patients with free-hand surgery implants [83]. The results of several randomized controlled trials show that implant with guided surgery leads to greater accuracy, less pain and swelling, and shorter surgery time, but is more costly than free-hand implants. In these studies, no significant differences were reported between guided implant surgery and free-hand surgery in terms of implant success/failure or clinical parameters such as marginal bone loss [84-86].

In a prospective cohort study, the clinical performance of guided implant surgery was evaluated in comparison with the free-hand method, and it was reported that implants performed using the surgical guide method increase the accuracy of implant placement. Also, angular deviation was one of the most important parameters improved using this method compared to the free-hand method [22].

\section{Advantages and Disadvantages of Free-Hand Surgery and Guided Surgery}

The guided surgery has several advantages. Manual errors related to implant placement in this method are greatly reduced. Since, when using this method, the least intervention is done on the patient, therefore, problems after surgery are minimal and both the patient and the doctor will be calm in terms of psychological dimension [87]. Implants performed in this way are much more accurate and have higher safety, so the results of the implant will be predictable. From a hygienic point of view, because the implants are placed in the correct position, the oral health of this method is relatively guaranteed. This method greatly increases the survival of implant placement [88-90].

There are many benefits to using a surgery guide for the dentist; the surgery guide optimizes the location, angle, and depth of implants. One of the most serious complications of implant surgery that can be minimized with guided implant surgery is damage to important anatomical structures (sinuses, nerves, arteries, and teeth) [91]. It also provides the dentist with increased vision of the surgical site and easy access to flap exposure. This method will also be immensely helpful for beginners. Due to the reduction of implant surgery time and high success rate and no failure in this method, the overall cost is much lower than other methods. Surgical guide is the best method for full edentulous cases to place all implants in parallel and the best way to place multiple implants side by side in parallel. Avoiding the retraction of flaps and sutures will reduce postoperative pain, edema and bleeding, and immediate resumption of oral hygiene practices [92-94].

It may also be advantageous in comparison with conventional methods in patients with reduced bone quantity. In theory, the need for augmenting the residual bone may be eliminated or decreased by optimizing implant positioning in the available bone $[95,96]$. On the opposite side of view, the available evidence lacks data regarding the application of digitally guided implant procedures to reconstruct resorbed edentulous ridges [97].

The application of an image-guided protocol in cases with severely resorbed posterior maxillae has been suggested as a proper alternative to insert implants in a restricted quantity of bone [98]. Considering the most recent systematic review studies [99-101], only the mentioned study was able to propose such a technique to propose the computer-assisted surgery as a substitute to bone regeneration techniques. Not all residual ridges could be managed accordingly and without considering reconstructing them. This is especially an important issue in patients with severe horizontal and vertical bone loss where anatomical structures could limit the final position of the implant [97].

The disadvantages of this method are several. Among other things, after making the guide, if necessary, it will not be allowed to change during the surgery. Also, if there is a change in the tissue between the time of ordering and the implant installation, it will change the fit of the prosthesis and ultimately the function of the implant prosthesis. If the guide is not stabilized and drilling is intended to penetrate hard bone, producing torsional forces on the sleeves, guide dislocation can occur during surgery. Also, in this method, there are costs related to purchasing software and special tools and drills, as well as spending time learning the curves for the treating physician [16, 89, 90, 102-104].

The advantages of free-hand implant placement include eliminating the time required to prepare the guide and reducing the cost of making the guide [25]. There are some limitations of free-hand implantation. First, clinical judgments about implant placement will be based on visualization of the clinical condition through information provided by cast and radiography. The second limitation is the longer time of this method than the surgical guide method because free-hand implant placement requires thinking and planning. Another limitation of the free-hand method is that aligning multiple implants using the freehand technique is difficult, and the results are less predictable than surgical guides $[13,105]$. Therefore, human error in this method will be much greater. Increased recovery time, swelling, pain, and bleeding are other disadvantages of the free-hand technique [13]. 


\section{Conclusion}

Dental implants have been a viable treatment option for patients with dental defects since the 1980s [106]. Dental implants have attracted the attention of patients and dentists due to the lack of damage to adjacent teeth and features such as comfort, high level of health, beauty, and long-term stability. However, observing such things as the correct depth of the implants is very necessary in performing the implant correctly. Today, with the development of techniques such as computer aided design and rapid prototyping, the surgical guide model has become increasingly used in dentistry. The use of a surgical pattern gives surgeons the chance to transfer the preoperative plan to the surgical procedure before taking any practical action on the patient, thereby both minimizing the invasion and shortening the operation time [106].

It seems that the surgical guide to some extent increases the accuracy of implant placement. Guided implant surgery can lead to errors that have been identified as the most common mistakes, such as misinterpretation of the tomographic image or misprocessing, deviation of 0.1 to $0.2 \mathrm{~mm}$ in the construction of the surgical guide, and incorrect fixation of the guide with the consequences of displacement during surgery [6]. The number of adjacent implants is a major predictor of mesiodistal position and angular accuracy. In contrast, tooth position and tooth extraction time are significantly related to position accuracy. The doctor's experience and the width of the edentulous space are also important factors in the position of implant placement and angulation, respectively [107].

In general, based on the results of clinical information, it can be concluded that, in people who have adjacent teeth and need an implant, a free-hand implant is a good option for them, but in contrast, for people who have no teeth at all and need to have multiple implants, proper guided surgery should be used. Narrow spaces less than $11 \mathrm{~mm}$ between teeth could be more suitable for free-hand implant placement in only one implant cases due to changes in position and lower angle changes. However, there is a possibility of approaching the root of the adjacent tooth and requires greater care. Because the placement of the implant immediately after tooth extraction improves the accuracy of the mesiodistal position. Therefore, using guided surgery over free-hand implant is preferred. It is also recommended that, in cases with incomplete bone regeneration and fallen roots, for example, in lower mill teeth, guided surgery should be used to counteract the drill's tendency to move toward the newly formed bone. It is also recommended that, in cases with incomplete bone regeneration and fallen roots, for example, in lower-molar teeth, guided surgery should be used to counteract the drill's tendency to move toward the newly formed bone [108].

It should be noted that high accuracy of implant placement is essential for ideal results, and guided surgery significantly increases accuracy, but unfortunately, this method is not yet used exclusively. Some dentists prefer to perform the implant by the free-hand method, which seems to be due to insufficient information to choose the right method for the implant. Since both free-hand and guided implant surgeries have their own advantages and disadvantages, it is recommended that dentists to have more extensive studies in this field to increase their knowledge and understanding of the use of these two methods and to use any technique to get the best results. Finally, it is recommended that guided surgery be preferred over the free-hand method when there is a possibility of error.

\section{Data Availability}

The data used to support the findings of this study are available from the corresponding author upon request.

\section{Ethical Approval}

This article is a review and does not contain any studies with human or animal performed by any of the authors.

\section{Conflicts of Interest}

The authors declare that they have no conflicts of interest.

\section{References}

[1] J. Cosyn, R. Wessels, R. Garcia Cabeza, J. Ackerman, C. Eeckhout, and V. Christiaens, "Soft tissue metric parameters, methods and aesthetic indices in implant dentistry: a critical review," Clinical Oral Implants Research, vol. 32, no. 21, pp. 93-107, 2021.

[2] M. Yamada and H. Egusa, "Current bone substitutes for implant dentistry," Journal of prosthodontic research, vol. 62, no. 2, pp. 152-161, 2018.

[3] A. Guentsch, L. Sukhtankar, H. An, and P. G. Luepke, "Precision and trueness of implant placement with and without static surgical guides: an in vitro study," The Journal of Prosthetic Dentistry, vol. 126, no. 3, pp. 398-404, 2021.

[4] R. Vinci, M. Manacorda, R. Abundo et al., "Accuracy of edentulous computer-aided implant surgery as compared to virtual planning: a retrospective multicenter study," Journal of Clinical Medicine, vol. 9, no. 3, p. 774, 2020.

[5] W. Geng, C Liu, Y Su, J Li, and Y Zhou, "Accuracy of different types of computer-aided design/computer-aided manufacturing surgical guides for dental implant placement," International Journal of Clinical and Experimental Medicine, vol. 8, no. 6, pp. 8442-9, 2015.

[6] D. N. Tatakis, H. H. Chien, and A. O. Parashis, "Guided implant surgery risks and their prevention," Periodontology, vol. 81, no. 1, pp. 194-208, 2019.

[7] C. D. Evans and S. T. Chen, "Esthetic outcomes of immediate implant placements," Clinical Oral Implants Research, vol. 19, no. 1, pp. 73-80, 2008.

[8] J. Brief, D. Edinger, S. Hassfeld, and G. Eggers, "Accuracy of image-guided implantology," Clinical Oral Implants Research, vol. 16, no. 4, pp. 495-501, 2005.

[9] C. H. Hämmerle and D. Tarnow, "The etiology of hard-and soft-tissue deficiencies at dental implants: a narrative review," Journal of Clinical Periodontology, vol. 45, pp. S267S277, 2018.

[10] J. D'haese, J. Ackhurst, D. Wismeijer, B. H. De, and A. Tahmaseb, "Current state of the art of computer-guided implant surgery," Periodontology 2000, vol. 73, no. 1, pp. 121-133, 2017. 
[11] R. Nair, "Implant surgical guides are they useful?" Prosthet Implant Dent, vol. 1, no. 2, pp. 107-115, 2018.

[12] T. Spielau, U. Hauschild, and J. Katsoulis, "Computerassisted, template-guided immediate implant placement and loading in the mandible: a case report," BMC Oral Health, vol. 19, no. 1, pp. 55-59, 2019.

[13] W. Choi, B. C Nguyen, A Doan, S Girod, B Gaudilliere, and D Gaudilliere, "Freehand versus guided surgery: factors influencing accuracy of dental implant placement," Implant Dentistry, vol. 26, no. 4, pp. 500-509, 2017.

[14] A. H. Shahrasbi and C. A. Hansen, "Surgical oral radiographic guide with a removable component for implant placement," The Journal of Prosthetic Dentistry, vol. 87, no. 3, pp. 330-332, 2002.

[15] S. Gupta, N Patil, J Solanki, R Singh, and S Laller, "Oral implant imaging: a review," Malaysian Journal of Medical Sciences: MJMS, vol. 22, no. 3, pp. 7-17, 2015.

[16] T. Tasopoulos, P.-G. Foskolos, G. Kouveliotis, and I. Karoussis, "Computer-guided implant dentistry," in Innovative Perspectives in Oral and Maxillofacial SurgerySpringer, Manhattan, NY, USA, 2021.

[17] J. Gargallo-Albiol, S. Barootchi, O. Salomó-Coll, and H.-1. Wang, "Advantages and disadvantages of implant navigation surgery. A systematic review," Annals of Anatomy - Anatomischer Anzeiger, vol. 225, pp. 1-10, 2019.

[18] S. Storelli et al., "A digital workflow for computer-guided implant surgery integrating $\mathrm{CBCT}$, model scanning, and CAD/CAM for a complete edentulism implant-supported prosthesis: a technique procedure," International Journal of Computerized Dentistry, vol. 23, no. 3, pp. 269-279, 2020.

[19] W. Zhou, Z. Liu, L. Song, C.-l. Kuo, and D. M. Shafer, "Clinical factors affecting the accuracy of guided implant surgery-A systematic review and meta-analysis," Journal of Evidence-Based Dental Practice, vol. 18, no. 1, pp. 28-40, 2018.

[20] F. Younes, J. Cosyn, T. De Bruyckere, R. Cleymaet, E. Bouckaert, and A. Eghbali, "A randomized controlled study on the accuracy of free-handed, pilot-drill guided and fully guided implant surgery in partially edentulous patients," Journal of Clinical Periodontology, vol. 45, no. 6, pp. 721-732, 2018.

[21] T. Joda, W. Derksen, J. G. Wittneben, and S. Kuehl, "Static computer-aided implant surgery (s-CAIS) analysing patientreported outcome measures (PROMs), economics and surgical complications: a systematic review," Clinical Oral Implants Research, vol. 29, no. 16, pp. 359-373, 2018.

[22] M. S. Block, R. W. Emery, D. R. Cullum, and A. Sheikh, "Implant placement is more accurate using dynamic navigation," Journal of Oral and Maxillofacial Surgery, vol. 75, no. 7, pp. 1377-1386, 2017.

[23] P. Smitkarn, K. Subbalekha, N. Mattheos, and A. Pimkhaokham, "The accuracy of single-tooth implants placed using fully digital-guided surgery and freehand implant surgery," Journal of Clinical Periodontology, vol. 46, no. 9, pp. 949-957, 2019.

[24] K. M. D'Souza and M. A. Aras, "Types of implant surgical guides in dentistry: a review," Journal of Oral Implantology, vol. 38, no. 5, pp. 643-652, 2012.

[25] F. C. Yogui, F. R. Verri, J. M. de Luna Gomes, C. A. A. Lemos, R. S. Cruz, and E. P. Pellizzer, "Comparison between computer-guided and freehand dental implant placement surgery: a systematic review and meta-analysis," International Journal of Oral and Maxillofacial Surgery, vol. 50, no. 2, pp. 242-250, 2021.
[26] M. Wang, X. Zhang, Y. Li, and A. Mo, “The influence of different guided bone regeneration procedures on the contour of bone graft after wound closure: a retrospective cohort study," Materials, vol. 14, no. 3, p. 583, 2021.

[27] J. Weitz, H. Deppe, S. Stopp, T. Lueth, S. Mueller, and B. Hohlweg-Majert, "Accuracy of templates for navigated implantation made by rapid prototyping with DICOM datasets of cone beam computer tomography (CBCT)," Clinical Oral Investigations, vol. 15, no. 6, pp. 1001-1006, 2011.

[28] F. F. Todescan, M. M. Hayashi, L. R. Giugni, M. A. Bottino, and J. P. M. Tribst, "From denture to the final implantsupported prosthesis using a full-digital protocol: a dental technique," Oral, vol. 1, no. 4, pp. 332-339, 2021.

[29] O. Schubert, J. Schweiger, M. Stimmelmayr, E. Nold, and J.-F. Güth, "Digital implant planning and guided implant surgery - workflow and reliability," British Dental Journal, vol. 226, no. 2, pp. 101-108, 2019.

[30] C.-C. Lin, C.-Z. Wu, M.-S. Huang, C.-F. Huang, H.-C. Cheng, and D. P. Wang, "Fully digital workflow for planning static guided implant surgery: a prospective accuracy study," Journal of Clinical Medicine, vol. 9, no. 4, p. $980,2020$.

[31] C. İlhan, M. Dikmen, and E. Yüzbaşioğlu, "Accuracy and efficiency of digital implant planning and guided implant surgery: an update and review," Journal of Experimental and Clinical Medicine, vol. 38, 2021.

[32] F. Kernen, J Kramer, L Wanner, D Wismeijer, K Nelson, and T Flügge, "A review of virtual planning software for guided implant surgery - data import and visualization, drill guide design and manufacturing," BMC Oral Health, vol. 20, no. 1, pp. 251-310, 2020.

[33] T. J. Balshi and D. G. Garver, "Surgical guidestents for placement of implants," Journal of Oral and Maxillofacial Surgery, vol. 45, no. 5, pp. 463-465, 1987.

[34] R. D'haese, T. Vrombaut, G. Hommez, H. De Bruyn, and S. Vandeweghe, "Accuracy of guided implant surgery in the edentulous jaw using desktop 3D-printed mucosal supported guides," Journal of Clinical Medicine, vol. 10, no. 3, p. 391, 2021.

[35] K. C. Oh, J.-S. Shim, and J.-M. Park, "In vitro comparison between metal sleeve-free and metal sleeve-incorporated 3Dprinted computer-assisted implant surgical guides," Materials, vol. 14, no. 3, p. 615, 2021.

[36] S. D, "Surgical guides for dental implants; a suggested new classification," Journal of Dentistry and Oral Health, vol. 1, no. 1, pp. 1-5, 2019.

[37] L. J. Stumpel III, "Cast-based guided implant placement: a novel technique," The Journal of Prosthetic Dentistry, vol. 100, no. 1, pp. 61-69, 2008.

[38] R. Blustein, R Jackson, K Rotskoff, R. E Coy, and D Godar, "Use of splint material in the placement of implants," The International Journal of Oral \& Maxillofacial Implants, vol. 1, no. 1, pp. 47-49, 1986.

[39] M. J. Engelman, J. A. Sorensen, and P. Moy, "Optimum placement of osseointegrated implants," The Journal of Prosthetic Dentistry, vol. 59, no. 4, pp. 467-473, 1988.

[40] Y. Ahmed, J. Shetty, K. M. Shetty, M. Paiddoddi, and S. A. Ahamed, "Surgical guide techniques and treatment planning for dental implant placement," Dental Poster Journal, vol. 10, no. 1, pp. 1-2, 2021.

[41] L.-d. Wang, W. Ma, S. Fu et al., "Design and manufacture of dental-supported surgical guide for genioplasty," Journal of Dental Science, vol. 16, no. 1, pp. 417-423, 2021. 
[42] S. Nelogi, M. Nelogi, and K. Rachana, "Computed tomography guided mock up driven designing of implant surgical guide: a novel technique," Journal of Dental Implants, vol. 11, no. 1, p. 60, 2021.

[43] M. Hudieb, M. AlKhader, S. Mortaja, M. Abusamak, N. Wakabayashi, and S. Kasugai, "Impact of bone augmentation of facial bone defect around osseointegrated implant: a three dimensional finite element analysis," Dentistry Journal, vol. 9, no. 10, p. 114, 2021.

[44] D. P. Sarment, P. Sukovic, and N. Clinthorne, "Accuracy of implant placement with a stereolithographic surgical guide," The International Journal of Oral \& Maxillofacial Implants, vol. 18, no. 4, pp. 571-7, 2003.

[45] P. Kiatkroekkrai, C. Takolpuckdee, K. Subbalekha, N. Mattheos, and A. Pimkhaokham, "Accuracy of implant position when placed using static computer-assisted implant surgical guides manufactured with two different optical scanning techniques: a randomized clinical trial," International Journal of Oral and Maxillofacial Surgery, vol. 49, no. 3, pp. 377-383, 2020.

[46] O. Ozan, K. Orhan, and I. Turkyilmaz, "Correlation between bone density and angular deviation of implants placed using CT-generated surgical guides," Journal of Craniofacial Surgery, vol. 22, no. 5, pp. 1755-1761, 2011.

[47] G. Unsal, I. Turkyilmaz, and S. Lakhia, "Advantages and limitations of implant surgery with CAD/CAM surgical guides: a literature review," Journal of clinical and experimental dentistry, vol. 12, no. 4, pp. e409-e417, 2020.

[48] S. Schnutenhaus, M. Wagner, C. Edelmann, R. G. Luthardt, and H. Rudolph, "Factors influencing the accuracy of freehand implant placement: a prospective clinical study," Dentistry Journal, vol. 9, no. 5, p. 54, 2021.

[49] A. L. C. Pereira, V. R. Medeiros, and A. . d.F. P. Carreiro, "Influence of implant position on the accuracy of intraoral scanning in fully edentulous arches: a systematic review," The Journal of Prosthetic Dentistry, vol. 126, 2020.

[50] M. Kola, A. Shah, H. Khalil et al., "Surgical templates for dental implant Positioning;Current knowledge and clinical perspectives," Al-Azhar Dental Journal for Girls, vol. 4, no. 3, pp. 215-222, 2017.

[51] K. Lal, G. S. White, D. N. Morea, and R. F. Wright, "Use of stereolithographic templates for surgical and prosthodontic implant planning and placement. Part I. The concept," Journal of Prosthodontics, vol. 15, no. 1, pp. 51-58, 2006.

[52] M. C. Metzger, A. Rafii, B. Holhweg-Majert, A. M. Pham, and B. Strong, "Comparison of 4 registration strategies for computer-aided maxillofacial surgery," OtolaryngologyHead and Neck Surgery, vol. 137, no. 1, pp. 93-99, 2007.

[53] M. Naitoh, E. Ariji, S. Okumura, C. Ohsaki, K. Kurita, and T. Ishigami, "Can implants be correctly angulated based on surgical templates used for osseointegrated dental implants?" Clinical Oral Implants Research, vol. 11, no. 5, pp. 409-414, 2000.

[54] H. J. Nickenig and S. Eitner, "Reliability of implant placement after virtual planning of implant positions using cone beam CT data and surgical (guide) templates," Journal of cranio-maxillo-facial surgery: Official Publication of the European Association for Cranio-Maxillo-Facial Surgery, vol. 35, no. 4-5, pp. 207-211, 2007.

[55] T. Bobek and A. Gordon, "A Review of the Determinants of a Successful Endosseous Implants," OSF Preprints, Charlottesville, VA, USA, 2021.

[56] N. Abdelhay, S. Prasad, and M. P. Gibson, "Failure rates associated with guided versus non-guided dental implant placement: a systematic review and meta-analysis," $B D J$ open, vol. 7, no. 1, pp. 31-39, 2021.

[57] P. Henprasert, D. V. Dawson, T. El-Kerdani, X. Song, E. Couso-Queiruga, and J. A. Holloway, "Comparison of the accuracy of implant position using surgical guides fabricated by additive and subtractive techniques," Journal of Prosthodontics, vol. 29, no. 6, pp. 534-541, 2020.

[58] K. Lakshmi Priya, A. Kumar, and A. Jaideep Mahendra, "A methodical review on guided implant surgery," Annals of the Romanian Society for Cell Biology, vol. 24, pp. 262-268, 2020.

[59] S. Bagchi, "Different Imaging Techniques for Dental Implants," Int J Prev Clin Dent Res, vol. 5, 2018.

[60] C. BouSerhal, R. Jacobs, M. Quirynen, and D. Steenberghe, "Imaging technique selection for the preoperative planning of oral implants: a review of the literature," Clinical Implant Dentistry and Related Research, vol. 4, no. 3, pp. 156-172, 2002.

[61] M. Andreiotelli, P. Kamposiora, and G. Papavasiliou, "Digital data management for CAD/CAM technology. An update of current systems," The European Journal of Prosthodontics and Restorative Dentistry, vol. 21, no. 1, pp. 9-15, 2013.

[62] N. R. Vaid, "Digital technologies in orthodontics-An update," Seminars in Orthodontics, vol. 24, 2018.

[63] S. Raikar, P. Talukdar, S. Kumari, S. Panda, V. Oommen, and A. Prasad, "Factors affecting the survival rate of dental implants: a retrospective study," Journal of International Society of Preventive and Community Dentistry, vol. 7, no. 6, p. 351,2017

[64] S. Olate, M. C. N. Lyrio, M. de Moraes, R. Mazzonetto, and R. W. F. Moreira, "Influence of diameter and length of implant on early dental implant failure," Journal of Oral and Maxillofacial Surgery, vol. 68, no. 2, pp. 414-419, 2010.

[65] D. Buser, L. Sennerby, and H. De Bruyn, "Modern implant dentistry based on osseointegration: 50 years of progress, current trends and open questions," Periodontology 2000, vol. 73, no. 1, pp. 7-21, 2017.

[66] K. Sagar, B. Subramony, S. Cheena, and G. Bhumika, "Dental Implant Failure Causes, Treatment, and Diagnosis: A Literature Review," Ahead of Print, 2021.

[67] S.-L. Oh, H. J. Shiau, and M. A. Reynolds, "Survival of dental implants at sites after implant failure: a systematic review," The Journal of Prosthetic Dentistry, vol. 123, no. 1, pp. 54-60, 2020.

[68] B. R. Chrcanovic, T. Albrektsson, and A. Wennerberg, "Reasons for failures of oral implants," Journal of Oral Rehabilitation, vol. 41, no. 6, pp. 443-476, 2014.

[69] B. R. Chrcanovic, J. Kisch, T. Albrektsson, and A. Wennerberg, "A retrospective study on clinical and radiological outcomes of oral implants in patients followed up for a minimum of 20 years," Clinical Implant Dentistry and Related Research, vol. 20, no. 2, pp. 199-207, 2018.

[70] M. Fathima, N. Sinha, and S. M. Ali, "Failures in dental implants: a review," Int J Adv Health Sci, vol. 4, no. 2, pp. 5-9, 2017.

[71] G. Tabanella, H. Nowzari, and J. Slots, "Clinical and microbiological determinants of ailing dental implants," Clinical Implant Dentistry and Related Research, vol. 11, no. 1, pp. 24-36, 2009.

[72] T. Fretwurst, K. Nelson, D. P. Tarnow, H.-L. Wang, and W. V. Giannobile, "Is metal particle release associated with peri-implant bone destruction? An emerging concept," Journal of Dental Research, vol. 97, no. 3, pp. 259-265, 2018. 
[73] D. Schneider, P. Marquardt, M. Zwahlen, and R. E. Jung, “A systematic review on the accuracy and the clinical outcome of computer-guided template-based implant dentistry," Clinical Oral Implants Research, vol. 20, pp. 73-86, 2009.

[74] G. Lin, S. Ye, F. Liu, and F. He, "A retrospective study of 30,959 implants: risk factors associated with early and late implant loss," Journal of Clinical Periodontology, vol. 45, no. 6 , pp. 733-743, 2018.

[75] P. K. Moy, D Medina, V Shetty, and T. L Aghaloo, "Dental implant failure rates and associated risk factors," The International Journal of Oral \& Maxillofacial Implants, vol. 20, no. 4, pp. 569-77, 2005.

[76] N. E. McDermott, S. K Chuang, V. V Woo, and T. B Dodson, "Complications of dental implants: identification, frequency, and associated risk factors," The International Journal of Oral \& Maxillofacial Implants, vol. 18, no. 6, pp. 848-55, 2003.

[77] Y.-H. Jung, B.-H. Cho, and J. J. Hwang, "Comparison of panoramic radiography and cone-beam computed tomography for assessing radiographic signs indicating root protrusion into the maxillary sinus," Imaging Science in Dentistry, vol. 50, no. 4, p. 309, 2020.

[78] M. S. Reddy, T. Mayfield-donahoo, F. J. J. Vanderven, and M. K. Jeffcoat, "A comparison of the diagnostic advantages of panoramic radiography and computed tomography scanning for placement of root form dental implants," Clinical Oral Implants Research, vol. 5, no. 4, pp. 229-238, 1994.

[79] M. R. Bueno, C. Estrela, B. C. Azevedo, and A. Diogenes, "Development of a new cone-beam computed tomography software for endodontic diagnosis," Brazilian Dental Journal, vol. 29, no. 6, pp. 517-529, 2018.

[80] E. Moudi, S. Haghanifar, K. Kazemian, R. Foroughi, M. Nabahti, and A. Bijani, "A comparative evaluation of cone-beam computed tomography and multi-slice computed tomography in the diagnosis of sheep mandibular bone defect: an in vitro study," Capian Journal Of Dental Research, vol. 7, no. 2, pp. 24-29, 2018.

[81] G. Frisardi, G Chessa, S Barone, A Paoli, A Razionale, and F Frisardi, "Integration of 3D anatomical data obtained by CT imaging and 3D optical scanning for computer aided implant surgery," BMC Medical Imaging, vol. 11, no. 1, pp. 5-7, 2011.

[82] D. Steenberghe, R. Glauser, U. Blomback et al., “A computed tomographic scan-derived customized surgical template and fixed prosthesis for flapless surgery and immediate loading of implants in fully edentulous maxillae: a prospective multicenter study," Clinical Implant Dentistry and Related Research, vol. 7, no. 1, pp. s111-s120, 2005.

[83] V. Arısan, C. Z. Karabuda, and T. Özdemir, "Implant surgery using bone-and mucosa-supported stereolithographic guides in totally edentulous jaws: surgical and post-operative outcomes of computer-aided vs. standard techniques," Clinical Oral Implants Research, vol. 21, no. 9, pp. 980-988, 2010.

[84] A. Pozzi, M Tallarico, M Marchetti, B Scarfò, and M Esposito, "Computer-guided versus free-hand placement of immediately loaded dental implants: 1-year post-loading results of a multicentre randomised controlled trial," European Journal of Oral Implantology, vol. 7, no. 3, pp. 229-242, 2014.

[85] M. Vercruyssen, C. Cox, W. Coucke, I. Naert, R. Jacobs, and M. Quirynen, "A randomized clinical trial comparing guided implant surgery (bone- or mucosa-supported) with mental navigation or the use of a pilot-drill template," Journal of Clinical Periodontology, vol. 41, no. 7, pp. 717-723, 2014.

[86] M. Vercruyssen, G. van de Wiele, W. Teughels, I. Naert, R. Jacobs, and M. Quirynen, "Implant- and patient-centred outcomes of guided surgery, a 1-year follow-up: an RCT comparing guided surgery with conventional implant placement," Journal of Clinical Periodontology, vol. 41, no. 12, pp. 1154-1160, 2014.

[87] T. Umapathy, B. Anila, C. Ashwini, and C. Jayam, "Overview of surgical guides for implant therapy," Journal of Dental Implants, vol. 5, no. 1, p. 48, 2015.

[88] D. Harris and M. Quirynene, "Guidelines for the Use of Diagnostic Imaging in Implant Dentistry," Update of the EAO Clinical Oral Implants Research, vol. 23, 2012.

[89] R. M. Meffert, Reconstructive Aesthetic Implant Surgery, LWW, Pennsylvania, USA, 2003.

[90] Y.-J. Huh, B.-R. Choi, K.-H. Huh et al., "In-vitro study on the accuracy of a simple-design CT-guided stent for dental implants," Imaging science in dentistry, vol. 42, no. 3, pp. 139-146, 2012.

[91] S. S. Khanna, S. M. Balaji, M. B. Priyadarshani, S. Syed, T. T. Syeda, and S. Mohammed, "Surgical Guides in Implants: A Review," Saudi Journal of Oral and Dental Research, 2020.

[92] W. Becker, M. Goldstein, B. E. Becker, L. Sennerby, D. Kois, and P. Hujoel, "Minimally invasive flapless implant placement: follow-up results from a multicenter study," Journal of Periodontology, vol. 80, no. 2, pp. 347-352, 2009.

[93] L. Sravan, N. Nandini, G. Kaur, M. M. Kshirsagar, N. Kumari, and R. Mishra, "Comparison of flapless technique and conventional flap technique during dental implant placement," Journal of Advanced Medical and Dental Sciences Research, vol. 8, no. 8, pp. 168-171, 2020.

[94] T. Fortin, J. L Bosson, M Isidori, and E Blanchet, "Effect of flapless surgery on pain experienced in implant placement using an image-guided system," The International Journal of Oral \& Maxillofacial Implants, vol. 21, no. 2, pp. 298-304, 2006.

[95] M. Beretta, P. P. Poli, S. Tansella, and C. Maiorana, "Virtually guided alveolar ridge reduction combined with computeraided implant placement for a bimaxillary implant-supported rehabilitation: a clinical report," The Journal of Prosthetic Dentistry, vol. 120, no. 2, pp. 168-172, 2018.

[96] M. Beretta, P. P. Poli, S. Tansella, M. Aguzzi, A. Meoli, and C. Maiorana, "Cast-free digital workflow for implant-supported rehabilitation in a completely edentulous patient: a clinical report," The Journal of Prosthetic Dentistry, vol. 125, no. 2, pp. 197-203, 2021.

[97] J.-S. Kang, S.-A. Kim, Y.-R. Heo, and M.-K. Son, "Digitally guided surgery and prosthesis with narrow implants in the anterior region," Oral Biology Research, vol. 45, no. 1, pp. 29-40, 2021.

[98] T. Fortin, M. Isidori, and H. Bouchet, "Placement of posterior maxillary implants in partially edentulous patients with severe bone deficiency using CAD/CAM guidance to avoid sinus grafting: a clinical report of procedure," The International Journal of Oral \& Maxillofacial Implants, vol. 24, no. 1, pp. 96-102, 2009.

[99] M. Hultin, K. G. Svensson, and M. Trulsson, "Clinical advantages of computer-guided implant placement: a systematic review," Clinical Oral Implants Research, vol. 23, no. s6, pp. 124-135, 2012.

[100] M. Vercruyssen, M. Hultin, A. N. Van, K. Svensson, I. Naert, and M. Quirynen, "Guided surgery: accuracy and efficacy," Periodontology 2000, vol. 66, no. 1, pp. 228-246, 2014.

[101] M. Vercruyssen, I. Laleman, R. Jacobs, and M. Quirynen, "Computer-supported implant planning and guided surgery: 
a narrative review," Clinical Oral Implants Research, vol. 26, no. S11, pp. 69-76, 2015.

[102] Y.-W. Chen, B. W. Hanak, T. C. Yang et al., "ComputerAssisted Surgery in Medical and Dental Applications," Expert Review of Medical Devices, vol. 18, 2021.

[103] T. J. Balshi, S. F Balshi, R Jaffin, M. A Salama, R. G Triplett, and S Parel, "CT-generated surgical guides and flapless surgery," The International Journal of Oral \& Maxillofacial Implants, vol. 23, no. 2, pp. 190-197, 2008.

[104] B. S. Chong, M Dhesi, and J Makdissi, "Computer-aided dynamic navigation: a novel method for guided endodontics," Quintessence International, vol. 50, no. 3, pp. 196-202, 2019.

[105] T. Tang, Z. Huang, L. Liao, X. Gu, J. Zhang, and X. Zhang, "Factors that influence direction deviation in freehand implant placement," Journal of Prosthodontics, vol. 28, no. 5, pp. 511-518, 2019.

[106] R. Noharet, A. Pettersson, and D. Bourgeois, "Accuracy of implant placement in the posterior maxilla as related to 2 types of surgical guides: a pilot study in the human cadaver," The Journal of Prosthetic Dentistry, vol. 112, no. 3, pp. 526532, 2014.

[107] S. Kühl, S. Zürcher, T. Mahid, M. Müller-Gerbl, A. Filippi, and P. Cattin, "Accuracy of full guided vs. half-guided implant surgery," Clinical Oral Implants Research, vol. 24, no. 7, pp. 763-769, 2013.

[108] M. Payer, R. Kirmeier, N. Jakse, C. Pertl, W. Wegscheider, and M. Lorenzoni, "Surgical factors influencing mesiodistal implant angulation," Clinical Oral Implants Research, vol. 19, no. 3, pp. 265-270, 2008. 